\title{
The Study and Implementation of Text-to-Speech System for Agricultural Information
}

\author{
Huoguo Zheng ${ }^{1,2, *}$, Haiyan $\mathrm{Hu}^{1,2}$, Shihong Liu ${ }^{1,2}$, and Hong Meng ${ }^{1,2}$ \\ ${ }^{1}$ Agricultural Information Institute, Chinese Academy of Agricultural Sciences, \\ Beijing, P.R. China 100081, \\ Tel.: +86-10-82106263; Fax: +86-10-82106263 \\ huoguos@caas.net.cn \\ ${ }^{2}$ Key Laboratory of Digital Agricultural Early-warning Technology, \\ Ministry of Agriculture, The People's Republic of China, Beijing, P.R. China 100081
}

\begin{abstract}
The Broadcast and Television coverage has increased to more than $98 \%$ in china. Information services by radio have wide coverage, low cost, easy-to-grass-roots farmers to accept etc. characteristics. In order to play the better role of broadcast information service, as well as aim at the problem of lack of information resource in rural, we R \& D the text-to-speech system. The system includes two parts, software and hardware device, both of them can translate text into audio file. The software subsystem was implemented basic on third-part middleware, and the hardware subsystem was realized with microelectronics technology. Results indicate that the hardware is better than software. The system has been applied in huailai city hebei province, which has conversed more than 8000 audio files as programming materials for the local radio station.
\end{abstract}

Keywords: text-to-speech, software, hardware device, microelectronics.

\section{Introduction}

After year's development, China has made the amazing progress in the development face the farmer's information service. Agricultural information service played a more positive role in solving the problem of "Farmers, Rural Areas and Agriculture Production" (Guo Hongmin et al, 2007). In the recent two years, there had emerged a number of new agriculture information service patterns, which providing timely and accurate information for farmers by the media of TV, phone and network, such as integrated service pattern in Hebei province (Gao Jikui et al, 2005), farmer's mail in Zhejiang province (Wu Yitian et al, 2007), given number "12316" in Jilin province (Jilin farmer website, 2007), 110 in science and technology in Hainan province (Wu Yuanbin et al, 2007). However, we should see that the radio and TV are still playing the main role of information service infrastructure in rural. According to the latest statistics, China's radio and television coverage has increased to more than $98 \%$.

\footnotetext{
* Corresponding author.
} 
Compared with other services, broadcasting as a carrier of information services has a wide coverage and low cost, easy-to-grass-roots farmers to accept and so on.

In the investigation and study we discovered that broadcasts the information service to emerge quietly in many places and the prospects for development are huge. To develop the broadcast information service well, we have researched and developed the rural broadcast information service system, including the network information acquisition, text-to-speech, the program arrangement; provide the information resource for the rural Broadcasting station. This article introduced the realization of text-to-speech system, which can transform the text information into the audio document, and provide program resource.

\section{Design of Text-to-Speech System}

Text-to-speech system, also known as text speech synthesis system, concludes two sub-systems, TTS software and TTS hardware. The system's functions are shown in Figure 1. Both of them can construct audio database by means of translating the text user defined or export from database into audio.

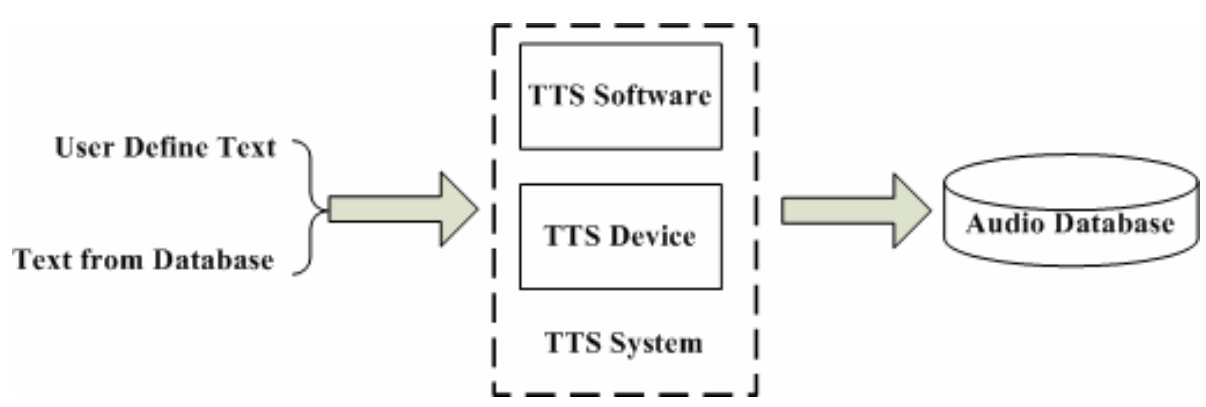

Fig. 1. Text-to-speech system's functions

\subsection{Design of TTS Software}

TTS software, which was implemented base on the third party middleware, can translate the user-defined text, text export from database into audio files. Its core function includes parameter setting, text-input, text-pretreatment and Text-to-Speech.

\subsection{Design of TTS Hardware}

TTS hardware sub-system includes the text speech synthesis hardware and the text-editing software. The synthesis hardware is overall system's core, which function is transforms the text information into the pronunciation, the text-editing software is the systematic input interface, which sent the text message to the hardware. Hardware must be associated with the computer through the USB interface to get power and adoption of the text-editing software to achieve text waiting to be converted between PC and hardware. 


\section{Design of hardware}

The hardware is the text-to-speech sub-system's foundation; its functions must be careful, comprehensive low-level hardware support. The hardware's connection principle is shown in Figure 2. The text speech synthesis hardware's main component include 8 microprocessor ATmega128 (Li Hua et al, 2008), the USB main line general connection chip, the XF-S4240 module, the low voltage audio power amplifier.

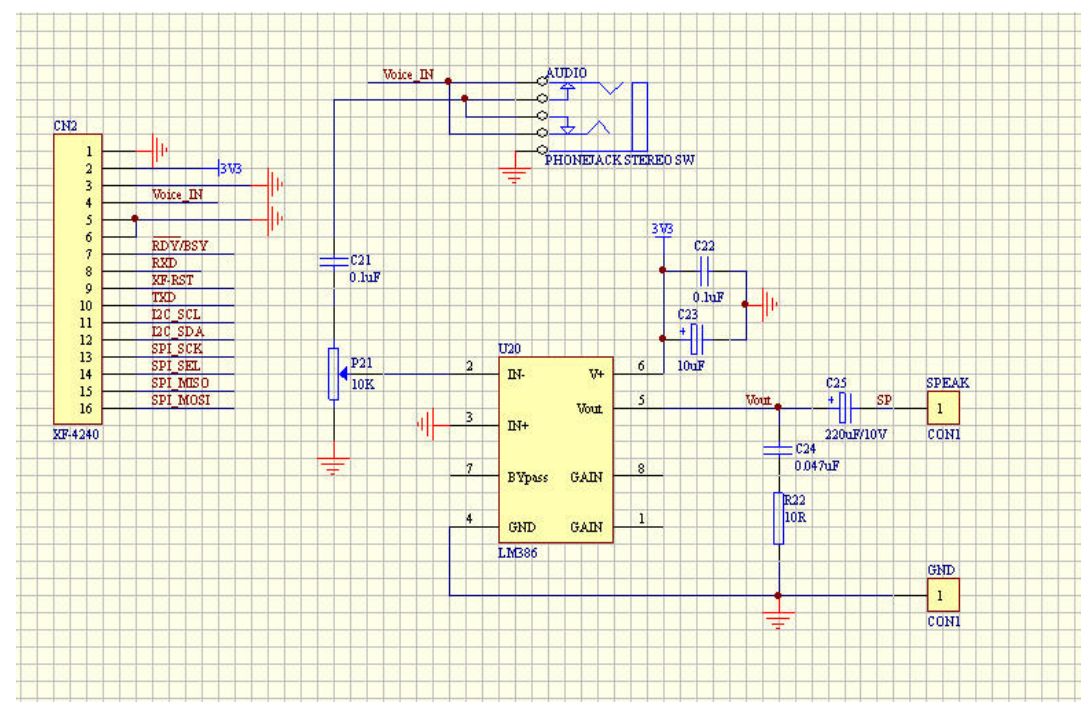

Fig. 2. The principle figure of text speech synthesis hardware

\section{Design of text-editing software}

The text-editing software is the controller of text data transmission between PC machine and the text speech synthesis hardware. After the process of edition, operation, pretreatment, the text can be accurately loaded, recognized, read by the outside loudspeaker. The test-editing software's major function includes: imports the text document (the .txt form), set special tags, preprocess the document, save the file and play voice. The text adopted by the special tags settings, you can effectively control the speed of play voice, tone, volume and so on, then have a clear, natural, accurate text-to-speech effect.

\section{Implementation of Text-to-Speech System}

\subsection{Implementation of Text-to-Speech Software}

The text-to-speech software has implemented the following functions, text-edit, audition, audition pause, audition stop, save the text as file and translate the text into audio file, base on the object-oriented software design thought, as well as the consideration of the interface of the software. The work flow of the software is shown in Figure 3. 


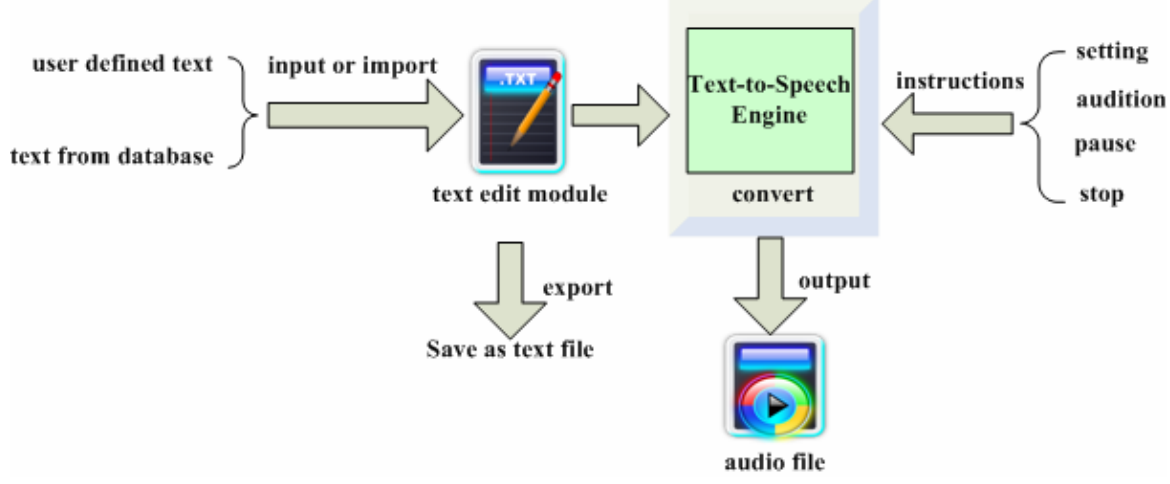

Fig. 3. The work flow of the text-to-speech software

\subsection{Implementation of Text-to-Speech Hardware}

\section{Implementation of software}

The hardware platform is composed of four components: the microprocessor, USB interface, the text speech synthesis module, as well as audio player. The hardware gets power and the text data by the USB interface from the computer, undergoes an 8-bit microprocessor system programming processing, to the information which receives carries on the correct judgment, the recognition again through the XF-S4240 text speech synthesis module, finally play the voice through the audio amplifier module. The audio can be recorded, preserved. The text speech synthesis hardware's work flow is shown in figure 4.

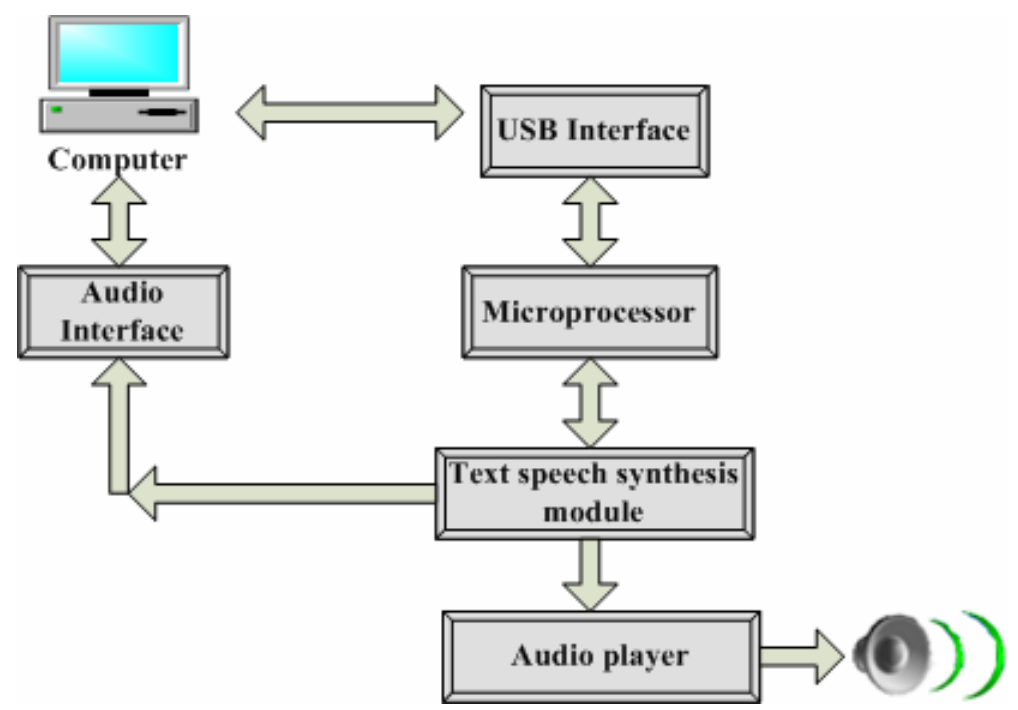

Fig. 4. The work flow of text speech synthesis module 
The single-chip embedded program realized by ICCAVR (Ma Chao et al, 2007) programming in the course of hardware programming (Qin Changjiang et al, 2007), and its function is getting text data through USB interface by means of interrupts, then sent the text data to XF-S4240 module to synthesis pronunciation, in addition gains the transition status in interrupt service through the serial port, back to the PC machine in order to control the continued transmission of data.

\section{Implementation of text-editing software}

Basic on the detailed analysis of whole text-to-speech system, we carry the work of design and realization on the text-editing software. According to the characteristic of the function, divides it into four modules: import text file, edit the tags, text speech synthesis and save the audio file. The text-editing software's work flow is shown in figure 5 .

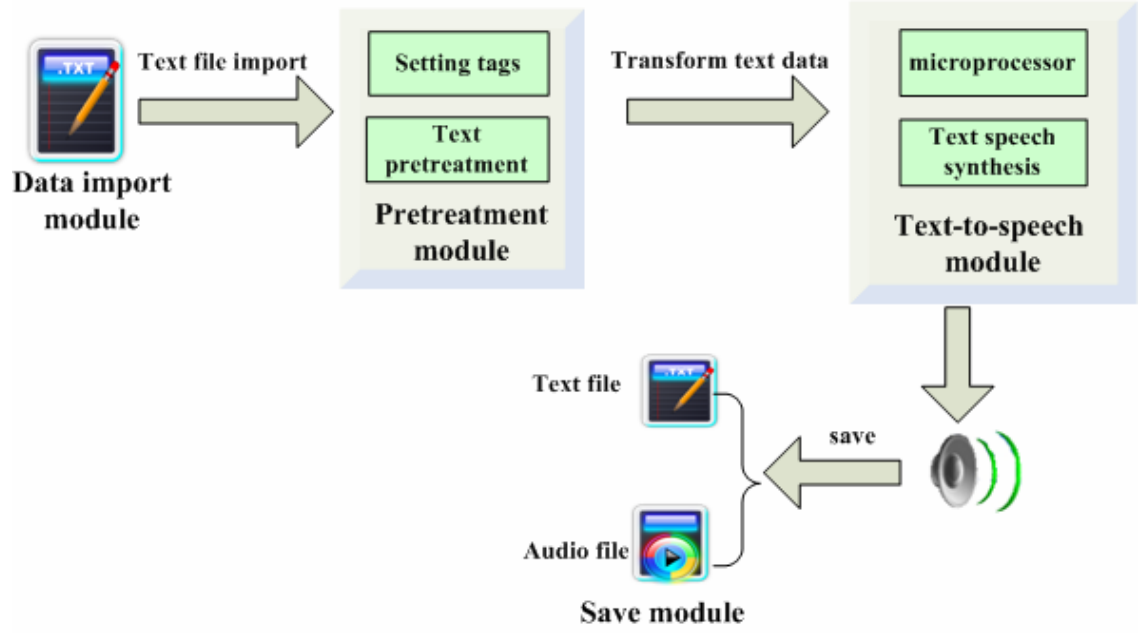

Fig. 5. The work flow of text-editing software

While the text-to-speech system running, we input or import the text which will be deal into the pretreatment module of the text-editing software, set format tags and pre-standardized, and then sent the processed text to the hardware, to judge the accuracy, identification. Finally, the text will be converted to voice, then play.

\section{Discussions}

Results indicate that the hardware works better than software. The audio file transformed by hardware sub-system is smoother than software sub-system. The text-to-speech system has translated more than 8000 piece of agricultural science and technology information into audio file in huailai radio station hebei province. Those audio files provide for the local farmers as information service by broadcast. Good effect achieved, according to the local people. 
China's rural reform has entered a new period of development. The effect of agriculture information service in the promotion of rural development, agricultural efficiency and increase farmers in rural appear already. Agriculture information will play a more and more important role in rural area. All sectors of society participate in the growing movement. Under this kind of advantageous environment, how to display the function of traditional media fully, innovate rural information service pattern, is the question which the scientific research worker and the department of information service must consider.

\section{References}

AIC Monaghan. Intonation in a text-to-speech conversion system. University of Edinburgh dissertation (1991)

Klatt, D.H.: Review of text-to-speech conversion for English. The Journal of the Acoustical Society of America (3), 737-743 (1990)

Hongmin, G., Yunhong, P.: Agriculture information service practice and discussion. Agricultural network Information 8(3) (2007)

Jikui, G., Yongqiang, L., Jianyu, C.: The three electricity and a hall information service system help the farmer be rich. China agricultural information (11) (2005)

Jilin farmer website. 12316 new rural hotline resolve famer's problem. Jilin agricultural and rural economic information (7) (2007)

Kain, A., Macon, M.W.: Spectral voice conversion for text-to-speech synthesis. In: Proceedings of the 1998 IEEE International Conference, vol. 1, pp. 285-288 (1998)

Hua, L., Wei, M., Baoyou, W.: Design of CAN-GPRS Gateway Based on ATMEGA128. Micro-computer information (3) (2008)

Lee, L.S., Tseng, C.Y., Ouh-Young, M.: The synthesis rules in a Chinese text-to-speech system. IEEE Transactions on Acoustics, Speech and Signal processing (9) (1989)

Chao, M.: AVR Monolithic integrated circuit embedded system principle and application practice. The Press of Beijing University of Aeronautics and Astronautics (2007)

Page, J.H., Breen, A.P.: The Laureate text-to-speech system: architecture and applications. BT technology journal (14), 57-67 (1996)

Changjiang, Q., Ziquan, Y., Yuquan, L.: Design and Implement of the Communication Bus Interface between PLD and AVR in VHDL. Micro-computer information, 6-2 (2008)

Yitian, W.: "farmer mailbox"- practice "new countryside" by means of informationization. The construction of informationization (3) (2007)

Yuanbin, W., Jinhua, W.: Agricultural science and technology 110 is the effective way to service the "Farmers, Rural Areas, Agriculture Production". Agricultural science and technology communication (1) (2007) 